\title{
Development of the In-Situ Ion Irradiation SEM at Sandia National Laboratories
}

Samuel A. Briggs ${ }^{1,2}$, Anthony Monterrosa ${ }^{1}$, Nathan Heckman ${ }^{1}$, Christopher M. Barr ${ }^{1}$, LaRico Treadwell ${ }^{1}$, Brad Boyce ${ }^{1}$, and Khalid Hattar ${ }^{1 *}$

1. Sandia National Laboratories, Albuquerque, NM 87123, USA

2. Oregon State University, Corvallis, OR 97331, USA

* Corresponding author: khattar@sandia.gov

Monitoring materials evolution in extreme environments using in-situ techniques allows for increased insight into the active mechanisms behind materials transformations and an enhanced understanding of the temporal dependencies of a given materials response. These types of experiments are especially useful for studying the combinatorial effects of super-imposed environmental stressors, such as those posed by nuclear reactor and radiation environments. The In-situ Ion Irradiation Transmission Electron Microscope (I ${ }^{3}$ TEM) at Sandia National Laboratories (SNL) was developed to study such microstructural changes in thin, electron transparent specimens [1]. The capability to study the effects of radiation damage, high temperatures, mechanical stresses, and other environmental variables has been demonstrated in several publications [2-4]. However, the $\mathrm{I}^{3} \mathrm{TEM}$ does not allow for the study of mesoscale and surface transformation, and in-situ TEM experiments in general have been known to be subject to various thin-film effects. As such, a complementary microscopy capability enabling work at larger length scales has been developed in the In-situ Ion Irradiation Scanning Electron Microscope $\left(\mathrm{I}^{3} \mathrm{SEM}\right)$ facility.

The I ${ }^{3}$ SEM facility mates a JEOL JSM-IT300HRLV FEG SEM with a 6 MV EN Tandem Van de Graaff-Pelletron accelerator. The SEM is equipped with the largest specimen chamber offered and is capable of $1.5 \mathrm{~nm}$ resolution and operating in low-vacuum modes with pressures up to $150 \mathrm{~Pa}$. The Tandem is coupled with four different ion sources (SNICS, Alphatross, duoplasmatron proton source, and Hiconex 834 sputter source) allowing for a wide range of ion species to be produced and directed into the SEM chamber at energies ranging from $800 \mathrm{keV}$ to $88 \mathrm{MeV}$. The two are mated via a custom adapter utilizing the WDS port of the stock JEOL SEM chamber. This port also allows for installation of a Kaufman \& Robinson KDC 10 gas-fed ion source capable of high-current ( $>10 \mathrm{~mA}$ ) implantation of $\mathrm{He}$ or other gaseous species with energies from less than $100 \mathrm{eV}$ to $1.2 \mathrm{keV}$. The Tandem and the KDC 10 source can be operated simultaneously for dual-beam experiments. While the secondary radiation produced during ion irradiation tends to saturate the secondary electron detectors, the ion beam can be interrupted for electron imaging, allowing for pseudo in-situ observation of microstructural evolution.

These ion irradiation capabilities can be paired with various in-situ SEM testing stages, such as the Hysitron PI-85 picoindenter, the MTI-Fulham heating/straining tensile stage, and a custom-built piezofatigue stage $[5,6]$, allowing for radiation creep, radiation fatigue, or simply high-temperature irradiation experiments to be performed. The SEM is also equipped with EDAX Velocity ${ }^{\mathrm{TM}}$ fast-scan EBSD camera and an EDAX Octane Elite silicon drift detector for EDS analysis, thus enabling studies on grain structure evolution or chemical segregation during pseudo in-situ irradiation. This suite of capabilities enables a plethora of mesoscale studies of materials behaviour in extreme environments.

Initial proof-of-concept experiments have been performed using $20 \mathrm{MeV} \mathrm{Au}{ }^{+}$ions into a boron-doped polymer. As demonstrated in Figure 2, the formation of ion-induced cracking on the surface of the 
polymer was observed in-situ. Experimental access to the $I^{3}$ SEM facility is available through both the Nuclear Science User Facilities (NSUF) and the Center for Integrated Nanotechnologies (CINT) user proposals [7].

\section{References:}

[1] K. Hattar, D. C. Bufford, \& D. L. Buller. Nuclear Instruments and Methods in Physics Research, Section B: Beam Interactions with Materials and Atoms, 338 (2014), p. 56-65.

[2] D. C. Bufford, S. H. Pratt, T. J. Boyle, \& K. Hattar. Chemical Communications, 50 (2014), p. 75937596.

[3] B. R. Muntifering, et al. Journal of Nuclear Materials, 482 (2016), p. 139-146.

[4] G. S. Jawaharram, et al. Scripta Materialia, 148 (2018), p. 1-4.

[5] T. A. Furnish, et al. Journal of Materials Science, 52 (2017), p. 46-59.

[6] N.M. Heckman, et al. Nanoscale, 10 (2018), 21231-21243

[7] This work was supported by the US Department of Energy, Office of Basic Energy Sciences. Sandia National Laboratories is a multi-mission laboratory managed and operated by National Technology and Engineering Solutions of Sandia, LLC., a wholly owned subsidiary of Honeywell International, Inc., for the U.S. Department of Energy's National Nuclear Security Administration under contract DE-NA0003525. SAND2019-1559 C

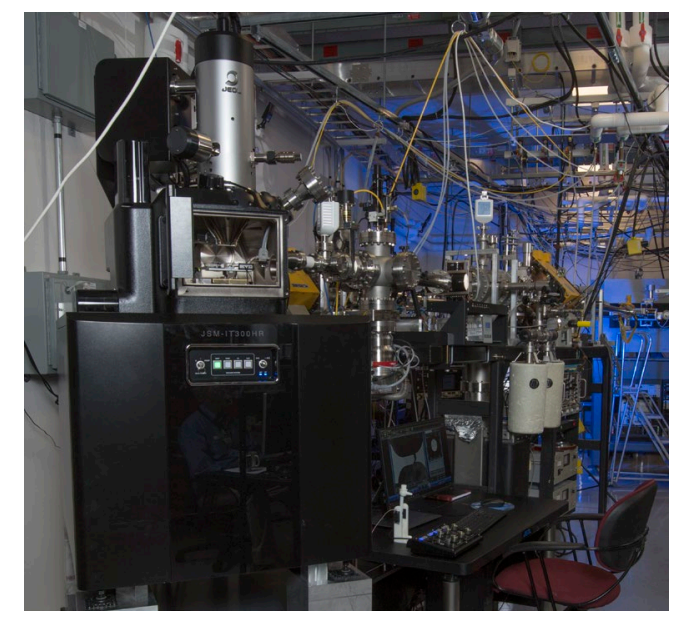

Figure 1. Image of the $I^{3} S E M$ facility at the Sandia National Laboratories Ion Beam Laboratory.

$$
\mathrm{t}=0 \mathrm{~s}
$$

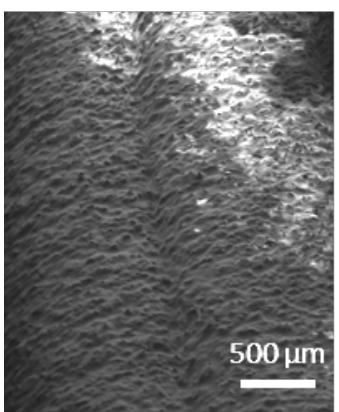

$t=720 s$

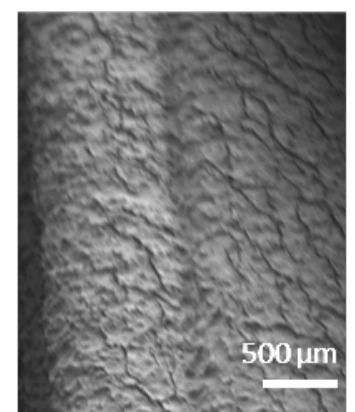

Figure 2. A boron-doped polymer imaged in the $I^{3}$ SEM before irradiation and after 720 seconds of irradiation with $20 \mathrm{MeV} \mathrm{Au}^{+}$ions. 